\title{
Design of Optimal Digital IIR Filters by Using a Bandwidth Adaptive Harmony Search Algorithm
}

\author{
Sayan Ghosh ${ }^{1}$, Debarati Kundu ${ }^{1}$, Kaushik Suresh ${ }^{1}$, Swagatam Das ${ }^{1}$, and Ajith Abraham ${ }^{2}$ \\ ${ }^{1}$ Dept. of Electronics and Telecommunication Engg, \\ Jadavpur University, Kolkata, India \\ 2 Norwegian University of Science and Technology, Norway \\ sayan88tito@gmail.com, kundu.debarati@gmail.com, kaushik_s1988@yahoo.com, ajith.abraham@ieee.org
}

\begin{abstract}
Evolutionary optimization algorithms have been recently applied to optimal digital IIR filter design. In this paper, we apply a Bandwidth Adaptive Harmony Search (BAHS) algorithm to the design of 1dimensional IIR filters. Harmony Search is an evolutionary algorithm, which emulates the improvisation process of musicians. We have modified the algorithm by setting the bandwidth equal to the standard deviation of the vectors in the harmony memory, and by introducing a penalty function to ensure IIR filter stability. The proposed variant has been applied to the design of low-pass, high-pass, band pass and band stop filters, by minimizing the $L_{1}$ and $L_{2}$ norm approximation errors and the maximum pass band and stop band ripples. The algorithm has been compared with the TIA approach. The harmony search variant has been found to outperform the TIA approach in designing IIR filters having near-desired frequency responses.
\end{abstract}

Index terms: Harmony search, Digital IIR filters, Metaheuristics,

\section{INTRODUCTION}

Evolutionary optimization algorithms have been recently successfully applied to the field of digital filter design[1-2]. IIR filter design is an important problem in this field. In IIR filters the design parameters are the coefficients of the different terms of the numerator and denominator in the filter transfer function. Given the frequency response of the desired filter, the optimization process involves finding the optimal design parameters to minimize, or maximize an objective function. The objective function describes the performance of the designed filter, and may take into account the desired response of the filter. There are different candidates for objective functions which describe the performance of the designed filter. Such objective functions may include the $L_{p}$ norm approximation errors, or the passband and stopband ripple magnitudes [3-5], or a weighted sum of both objectives. The goal of the optimization process is to find the optimal filter coefficients to closely approximate the desired filter response. The basic philosophy underlying evolutionary algorithms is that a population consisting of candidate solutions is initialized, and the agents cooperate with one another in trying to find the optimal point. One such algorithm, Harmony Search has recently emerged as a popular optimization algorithm. It simulates the improvisation process of musicians when they tune their instruments. The algorithm was introduced by Geem et al [6], and has been successfully applied to several real-life applications [7-8]. In this paper we introduce a variant of Harmony Search, called BAHS, which modifies the mutation process based on the variance of the harmony memory.

The rest of the paper is organized as follows: Section II deals with the general optimization process for digital IIR filters. Section III describes the basic harmony search algorithm and its modification. In Section IV, we apply the modified algorithm, along with the TIA approach [10] to the design of low-pass, high-pass, band pass and band stop filters. The results of the comparison are described in Section V, and are illustrated by convergence plots, frequency response plots, and pole-zero maps. Finally, in Section VI, we offer some concluding remarks.

\section{DESIGN OF DIGITAL IIR FILTERS}

The following recursive difference equation describes the design of an IIR filter:

$$
y(n)=\sum_{k=0}^{U} a_{k} x(n-k)-\sum_{k=0}^{V} b_{k} y(n-k)
$$

where $a_{k}$ and $b_{k}$ are filter coefficients, $x(n)$ and $y(n)$ are the input and output sequences, and $U$ and $V$ are the number of $a_{k}$ and $b_{k}$ filter coefficients where $V \geq U$. 
From the above difference equation, the general transfer function for an IIR filter is:

$$
H(z)=\frac{\sum_{k=0}^{U} a_{k} z^{-k}}{1+\sum_{k=1}^{V} b_{k} z^{-k}}
$$

The optimization process involves finding the optimal vector of filter coefficients $x=\left(a_{0}, a_{1}, a_{2}, \ldots, a_{U}, b_{1}, b_{2}, b_{3}, \ldots, b_{V}\right)$ so that the frequency response of the designed filter very closely approximates the desired response. The optimization problem is constrained in nature, since the filter must be BIBO (Bounded Input Bounded Output) stable. To ensure stability of the filter, all poles of $H(z)$ must lie within the unit circle $|z|=1$ in the $z$-plane. In this paper, we consider the particular case when $U=V$. If $x$ is the vector of filter coefficients for the designed filter $\mathrm{F}$, and $\omega$ is the digital angular frequency, then the $\mathrm{L}_{\mathrm{p}}$ norm approximation error is given by:

$L_{p}^{F}(x)=\left[\left.\int_{\Omega}\left|H_{I}(\omega)-\right| H_{F}(\omega, x)\right|^{p} d \omega\right]^{1 / p}$

$\Omega$ is the desired union of both passband and stopband, and $H_{I}(\omega)$ and $H_{F}(\omega, x)$ are, respectively the frequency responses of the desired and designed filters. $p$ is a real number in the range $[1, \infty]$. For a digital computer-based implementation, we quantize the digital angular frequency and replace the integration by a summation. This technique is called the fixed-grid-approximation.

The approximated value of the $L_{p}$ norm error is now given by:

$$
L_{p}^{F}(x)=\left[\left.\sum_{i=1}^{K}\left|H_{I}\left(\omega_{i}\right)-\right| H_{F}\left(\omega_{i}, x\right)\right|^{p}\right]^{1 / p}
$$

The range of $0 \leq \omega \leq \pi$ is quantized into $K$ equally spaced points, and the approximated value is calculated from the above summation.

$H_{I}\left(\omega_{i}\right)$ is defined to be:

$$
\begin{aligned}
H_{I}\left(\omega_{i}\right)=0 & & \omega_{i} \in \text { stopband } \\
=1 & & \omega_{i} \in \text { passband }
\end{aligned}
$$

$\delta_{1}$ and $\delta_{2}$ are the ripple magnitudes of passband and stopband, and are defined as :

$$
\delta_{1}=\max _{\omega_{i}}\left\{\left|H_{F}\left(\omega_{i}, x\right)\right|\right\}-\min _{\omega_{i}}\left\{\left|H_{F}\left(\omega_{i}, x\right)\right|\right\}
$$

when $\omega_{i} \in$ passband, and $\delta_{2}=\max _{\omega_{i}}\left\{\left|H_{F}\left(\omega_{i}, x\right)\right|\right\} \quad$ when $\omega_{i} \in$ stopband

The objective function used in our experiments is a weighted sum and is given by:

$$
\begin{aligned}
f(x)=w_{1} L_{1}^{F}(x)+ & w_{2} L_{2}^{F}(x) \\
& +w_{3} \delta_{1}(x)+w_{4} \delta_{2}(x)
\end{aligned}
$$

where $w_{1}, w_{2}, w_{3}$, and $w_{4}$ are suitable weight values.

\section{THE HARMONY SEARCH ALGORITHM}

Harmony Search is a population-based stochastic optimization algorithm which was introduced by Geem et.al. in [6] . Harmony Search employs musicinspired metaheuristics to find the vector for which the objective function has its global optima. Geem et al. in [6] observes that in harmony search, each dimension of the vector corresponds to a musical instrument, and the components along each dimension correspond to the notes played by each instrument. Subsequently, tuning each instrument is similar to producing a perturbation in each component of the vector.

The algorithmic steps of the basic Harmony Search Algorithm are given below:

1. Initialize the harmony memory by initializing $k$ random vectors uniformly throughout the search space range.

2 . Create a new vector $x^{\prime}$. For each component $x_{i}^{\prime}$ :

(a) with probability $p_{h m c r}$ pick the component from memory,

(b) with probability $1-p_{\text {hmcr }}$ pick a new random value in the allowed range.

3. Pitch adjustment: Only for component $x_{i}^{\prime}$ which was chosen from memory, with probability $p_{p a r}$ change $x_{i}^{\prime}$ by a small amount, called the distant bandwidth, and with probability $1-p_{p a r}$ do nothing.

4. If $x^{\prime}$ is better than the worst $x^{i}$ in the memory, then replace $x^{i}$ by $x^{\prime}$.

Repeat from step 2 until a maximum number of iterations has been performed.

Since the selection step may decrease the population variance, the variance operators must increase it necessarily. We propose a variant of Harmony search in which (1) $p_{h m c r}$ is set very close to unity, and (2) the distance bandwidth for each dimension is set equal to the standard deviation. In our proposed variant, we have followed modification (1) and changed modification (2), by setting the distance bandwidth equal to $+s$ or $-S$ with probability 0.5 . This promotes search capability. 
Table 1. Design specifications of the LP,HP,BP and BS filters

\begin{tabular}{|c|c|c|c|}
\hline $\begin{array}{c}\text { Filter } \\
\text { type }\end{array}$ & Passband & Stopband & $\begin{array}{c}\text { Maximum value of } \\
H_{F}\left(\omega_{i}, x\right)\end{array}$ \\
\hline LP & $0 \leq \omega \leq 0.2 \pi$ & $0.3 \pi \leq \omega \leq \pi$ & 1 \\
\hline HP & $0.8 \pi \leq \omega \leq \pi$ & $0 \leq \omega \leq 0.7 \pi$ & 1 \\
\hline BP & $0.4 \pi \leq \omega \leq 0.6 \pi$ & $\begin{array}{c}0 \leq \omega \leq 0.25 \pi \\
0.75 \pi \leq \omega \leq \pi\end{array}$ & 1 \\
\hline BS & $\begin{array}{c}0 \leq \omega \leq 0.25 \pi \\
0.75 \pi \leq \omega \leq \pi\end{array}$ & $0.4 \pi \leq \omega \leq 0.6 \pi$ & 1 \\
\hline
\end{tabular}

\section{IIR FILTER DESIGN USING HARMONY SEARCH}

We have applied the Bandwidth Adaptive Harmony Search (BAHS) algorithm to the field of IIR filter design, and we have compared it to the peformance of the TIA (Taguchi Immune Algorithm) approach to the same problem. Table 1 shows the desired frequency responses, and specifies the passband and stopband frequency ranges. The harmony memory contains the candidate coefficients of the IIR filter to be designed. Every vector in the harmony memory is a candidate solution to the optimization problem. Hence every vector to be of the form $\vec{x}=\left(a_{0}, a_{1}, a_{2}, \ldots, a_{U}, b_{1}, b_{2}, b_{3}, \ldots, b_{V}\right) \quad$ where $U=V=3$. Then the optimization problem is seven dimensional, since there are seven design parameters. Hence evolutionary algorithms can be effectively applied to this problem. The cost function returns the performance value of the vectors if the transfer function is stable, and returns a large penalty value, if the transfer function becomes unstable. This strategy of assigning a penalty value prevents the vectors from representing unstable filters. Transfer function stability is determined by checking if all poles lie inside the unit circle $|z|=1$ in the $z$-plane. The $\mathrm{L}_{\mathrm{p}}$ norm approximation errors have been evaluated for $p=1$ and $p=2$. In the computation of the $\mathrm{L}_{\mathrm{p}}$ norm errors, we have divided the frequency range $[0, \pi]$ into 200 equally spaced points. Then the number of points over which summation is performed are 182 for the low-pass and high-pass, and 143 for the bandpass and band stop filters respectively. The objective function is the same as defined in equation (2). The values of $w_{1}, w_{2}, w_{3}$ and $w_{4}$ are different.
Since the design should give paramount importance to the pass band and stop band ripple magnitudes, $w_{3}$ and $w_{4}$ have been set to larger values in comparison with $w_{1}$ and $w_{2}$. For the low-pass filter design, $w_{1}, w_{2}, w_{3}$ and $w_{4}$ are set to $1,1,8$ and 15 respectively. For the high-pass filter, $w_{1}, w_{2}, w_{3}$ and $w_{4}$ are set to $1,1,15$ and 8 respectively. $w_{1}$ $, w_{2}, w_{3}$ and $w_{4}$ are set to $1,1,20$ and 15 respectively for the band pass filter to to $1,1,20$ and 12 respectively for the band stop filter. The size of the harmony memory is set to 40, and the algorithms are run for 25 trials in each filter design experiment (LP, HP, BP or BS) where each trial uses 160,000 function evaluations. For each experiment we record the transfer function and frequency responses of the filter with the lowest objective function. The experiments discussed here have been performed in MATLAB environment on a Pentium IV, $2.3 \mathrm{GHz}$ $\mathrm{PC}$, with $1024 \mathrm{~KB}$ cache and $2 \mathrm{~GB}$ of main memory in Windows XP environment.

\section{RESULTS AND DISCUSSIONS}

Table 2 and 3 shows optimized values of the $L_{1}$ and $\mathrm{L}_{2}$ norm approximation errors, and the passband and stopband ripple magnitudes for the LP,HP,BP and BS filters designed by BHS and TIA approach respectively. From the table we note that the algorithm has been highly effective in the design of different types of filters, Figure 1 shows the frequency responses of the LP and HP filters, and Figure 2 and 3 show the frequency responses for BP and BS filters respectively. It is observed that the poles lie within the unit circle, thus illustrating the utility of the penalty scheme in the design of stable filters. 
Table 2: The minimum values of the $\mathrm{L}_{1}$ and $\mathrm{L}_{2}$ norm approximation errors, and the minimum values of passband and stopband ripples obtained using Bandwidth-Adaptive Harmony Search.

\begin{tabular}{|c|c|c|c|c|}
\hline Filter type & $\begin{array}{c}\mathrm{L}_{1} \text { norm approximation } \\
\text { error }\end{array}$ & $\begin{array}{c}\mathrm{L}_{2} \text { norm } \\
\text { approximation error }\end{array}$ & $\begin{array}{c}\text { Maximum } \\
\text { passband } \\
\text { ripple }\end{array}$ & Maximum stopband ripple \\
\hline LP & 5.7010 & 0.9374 & 0.0491 & 0.3457 \\
\hline HP & 3.9170 & 0.5957 & 0.0660 & 0.2245 \\
\hline BP & 5.5827 & 0.5813 & 0.0355 & 0.1402 \\
\hline BS & 4.2651 & 0.5087 & 0.0580 & 0.1260 \\
\hline
\end{tabular}

Table 3: The minimum values of the $\mathrm{L}_{1}$ and $\mathrm{L}_{2}$ norm approximation errors, and the minimum values of passband and stopband ripples obtained using TIA approach

\begin{tabular}{|c|c|c|c|c|}
\hline Filter type & $\begin{array}{c}\mathrm{L}_{1} \text { norm approximation } \\
\text { error }\end{array}$ & $\begin{array}{c}\mathrm{L}_{2} \text { norm } \\
\text { approximation error }\end{array}$ & $\begin{array}{c}\text { Maximum } \\
\text { passband } \\
\text { ripple }\end{array}$ & Maximum stopband ripple \\
\hline LP & 7.2754 & 0.9311 & 0.0778 & 0.3054 \\
\hline HP & 11.6301 & 1.4135 & 0.1063 & 0.4145 \\
\hline BP & 7.8782 & 0.7418 & 0.1454 & 0.1241 \\
\hline BS & 8.0120 & 0.7951 & 0.0967 & 0.1513 \\
\hline
\end{tabular}

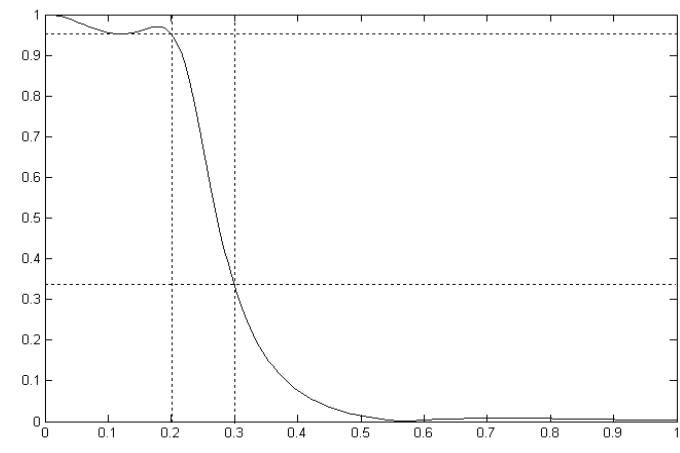

Fig. 1(a)

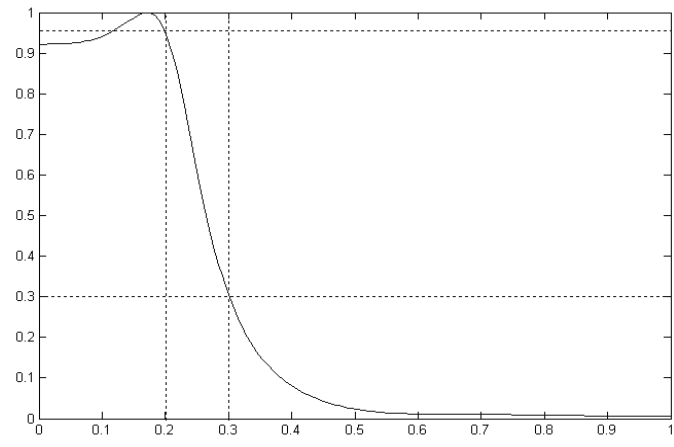

Fig. 1(b)

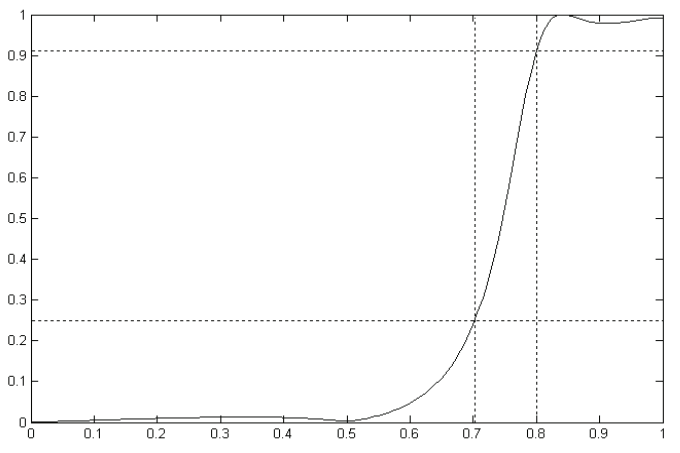

Fig. 1(c)

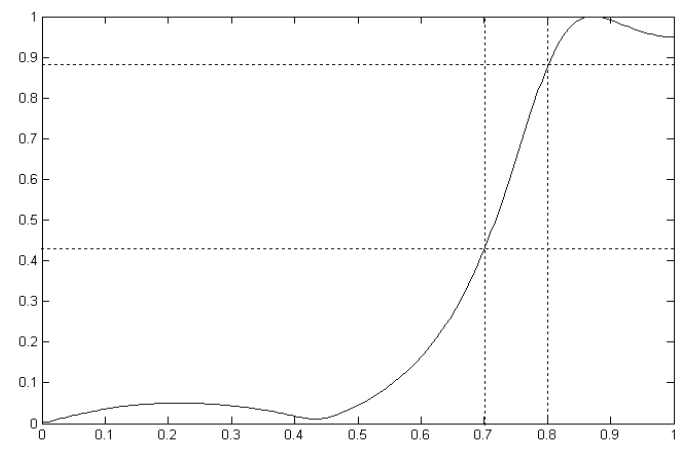

Fig. 1(d)

Figure 1. Frequency response plots as functions of normalized frequency for the Harmony Search (Fig.1 (a) and (c)) and TIA approaches (Fig.1 (b) and (d)) applied to LP and HP filter design. 
Table 2: The z-domain transfer functions of the LP,HP, BP and BS filters designed using the BAHS algorithm.

\begin{tabular}{|c|c|}
\hline $\begin{array}{c}\text { Filter } \\
\text { type }\end{array}$ & Transfer function \\
\hline LP & $H_{L P}(z)=0.0305 \frac{(z+1.3183)\left(z^{2}+0.4197 z+1.0124\right)}{(z-0.5996)\left(z^{2}-1.2260 z+0.6559\right)}$ \\
\hline HP & $H_{H P}(z)=0.0360 \frac{(z-1.0628)\left(z^{2}-0.0031 z+1.1083\right)}{(z-0.5753)\left(z^{2}+1.2893 z+0.6579\right)}$ \\
\hline $\mathrm{BP}$ & $H_{B P}(z)=0.1696 \frac{(z+1.07)(z-0.8523)\left(z^{2}+1.643 z+0.9685\right)\left(z^{2}-1.501 z+0.8838\right)}{(z+0.5813)(z-0.3314)\left(z^{2}+0.6058 z+0.5424\right)\left(z^{2}-0.8126 z+0.6499\right)}$ \\
\hline $\mathrm{BS}$ & $H_{B S}(z)=0.4028 \frac{(z+0.5396)(z-0.0757)\left(z^{2}-0.7146 z+1.037\right)\left(z^{2}+0.318 z+0.9526\right)}{(z+0.6381)(z-0.3961)\left(z^{2}+0.9242 z+0.5828\right)\left(z^{2}-1.016 z+0.71\right)}$ \\
\hline
\end{tabular}

Table 3: The z-domain transfer functions of the LP,HP, BP and BS filters designed using the TIA approach.

\begin{tabular}{|c|c|}
\hline $\begin{array}{c}\text { Filter } \\
\text { type }\end{array}$ & Transfer function \\
\hline LP & $H_{L P}(z)=0.0369 \frac{(z+1.4752)\left(z^{2}+0.2795 z+0.5485\right)}{(z-0.5265)\left(z^{2}-1.2643 z+0.6472\right)}$ \\
\hline $\mathrm{HP}$ & $H_{H P}(z)=0.1094 \frac{(z-1.02)\left(z^{2}-0.3826 z+0.8735\right)}{(z-0.5164)\left(z^{2}+1.133 z+0.478\right)}$ \\
\hline $\mathrm{BP}$ & $H_{B P}(z)=0.1608 \frac{(z+1.1945)(z-0.9066)\left(z^{2}+1.5432 z+0.8891\right)\left(z^{2}-1.4321 z+0.8976\right)}{(z+0.5512)(z-0.3014)\left(z^{2}+0.5578 z+0.4786\right)\left(z^{2}-0.7827 z+0.6578\right)}$ \\
\hline $\mathrm{BS}$ & $H_{B S}(z)=0.4078 \frac{(z-0.0645)(z+0.5864)\left(z^{2}+0.2876 z+0.9426\right)\left(z^{2}-0.7428 z+1.0186\right)}{(z-0.3564)(z+0.6254)\left(z^{2}+0.8845 z+0.5574\right)\left(z^{2}-0.9872 z+0.6613\right)}$ \\
\hline
\end{tabular}

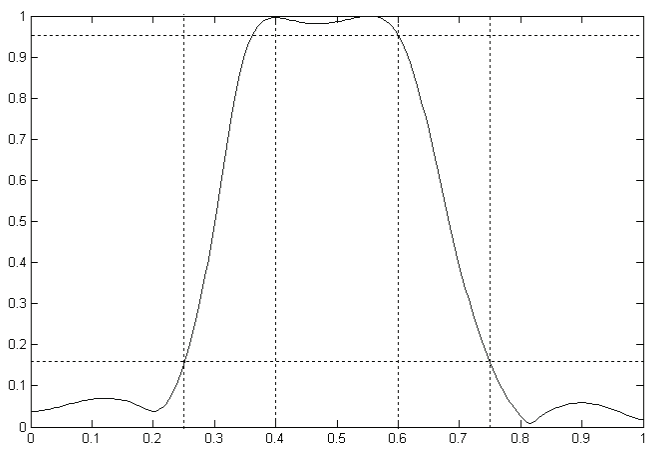

Fig. 2(a)

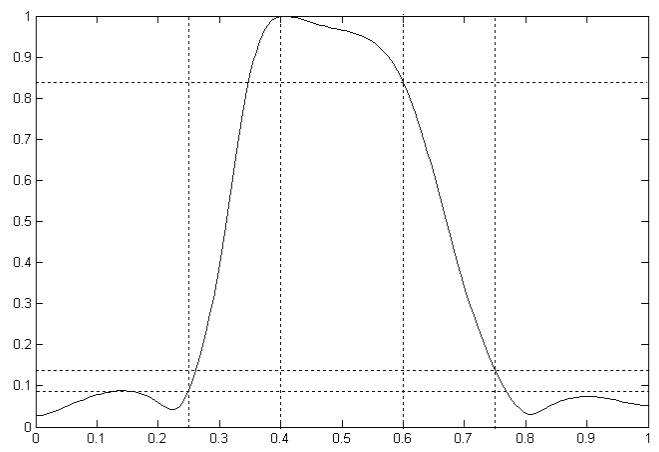

Fig. 2(b)

Figure 2. Frequency response plots as functions of normalized frequency for the Harmony Search (Fig.2 (a)) and TIA approaches (Fig.2 (b)) applied to BP filter design. 


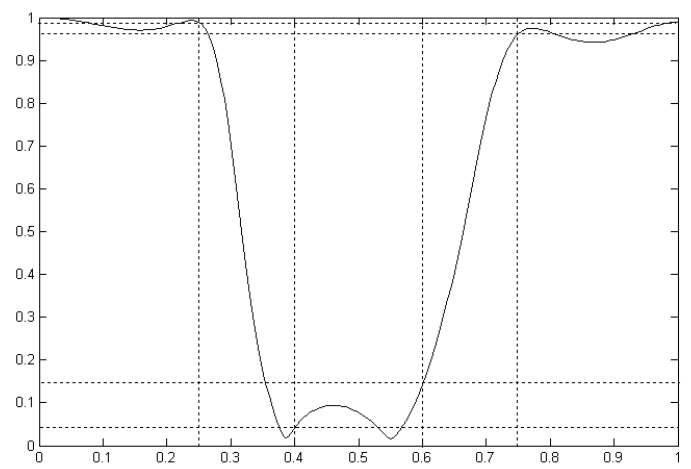

Fig. 3(a)

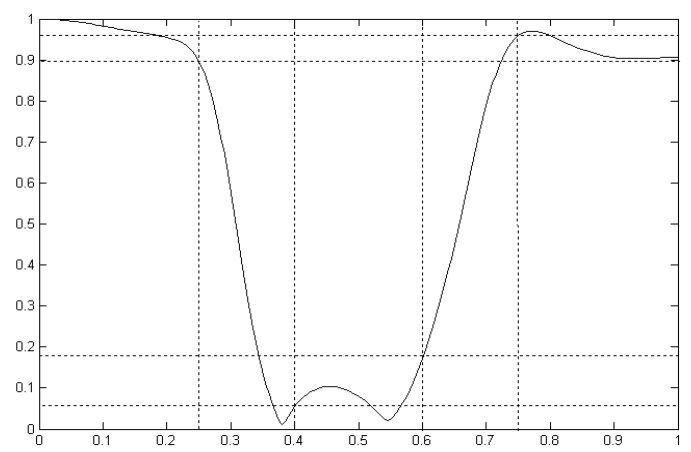

Fig. 3(b)

Figure 3. Frequency response plots as functions of normalized frequency for the Harmony Search (Fig.3 (a)) and TIA approaches (Fig.2 (b)) applied to BS filter design.

\section{CONCLUSIONS}

This paper has presented a new variant of the basic Harmony Search Algorithm for digital IIR filter design. The variant has been shown to be highly effective when applied to design optimal LP, HP ,BP and BS filters. The objective functions have been successfully optimized and reflect the trade-off between conflicting objectives. The frequency responses closely approximate the ideal filter responses. Through experiments, the proposed algorithm has been shown to be superior to the TIA approach in designing digital IIR filters. Future research may focus on the application of the BAHS algorithm to the design of other types of digital filters like FIR filters, differentiators and Hilbert Transformers [11].

\section{References}

[1] J. H. Li and F. L. Yin, "Genetic optimization algorithm for designing IIR digital filters," J. China Inst. Commun., vol. 17, pp. 1-7, 1996.

[2] S. C. Ng, C. Y. Chung, S. H. Leung, and A. Luk, "Fast convergent genetic search for adaptive IIR filtering," in Proc. IEEE Int. Conf. Acoust., Speech Signal Process., Adelaide, 1994, pp. $105-108$.

[3] A. G. Deczky, "Synthesis of recursive filters using the minimum p-error criterion," IEEE Trans. Audio Electroacoust., vol. 20, pp. 257-263, 1972.

[4] G. Cortelazzo and M. R. Lightner, "Simultaneous design in both magnitude and group-delay of IIR and FIR filters based on multiple criterion optimization," IEEE Trans. Acoust., Speech, Signal Process., vol. 32, pp. 949-967, 1984.

[5] M. Lang, "Optimal weighted phase equalization according to the L -norm," Signal Process., vol. 27, pp. 87-98, 1992.

[6] Z.Geem et al. "A New Heuristic Optimization Algorithm: Harmony Search”, Simulation, 2001.

[7] Z. Geem et al. "Harmony search optimization: Application to pipe network design.", International Journal of Modelling \& Simulation, 2002.

[8] A Vasebi, M Fesanghary, SMT Bathaee, "Combined heat and power economic dispatch by harmony search algorithm", International Journal of Electrical Power and Energy Systems, 2007 - Elsevier.

[9] R Forsati, AT Haghighat, M Mahdavi, "Harmony search based algorithms for bandwidth-delay-constrained least-cost multicast routing", Computer Communications, 2008 Elsevier.

[10] Jinn-Tsong Tsai and Jyh-Horng Chou, " Design of Optimal Digital IIR Filters by Using an Improved Immune Algorithm", IEEE Transactions on signal processing, vol. 54, no. 12, december 2006.

[11] IW Selesnick, "Optimal FIR and IIR Hilbert transformer design via LS and minimaxfitting", IEEE Transactions on signal processing, 2002. 